\title{
Effect of the three-dimensional microstructure on the sound absorption of foams: A parametric study
}

\author{
Fabien Chevillotte ${ }^{\mathrm{a})}$ \\ Matelys—Research Lab, Bât. B, 7 rue des maraîchers, F69120 Vaulx-en-Velin, France \\ Camille Perrot ${ }^{\text {b) }}$ \\ Université Paris-Est, Laboratoire Modélisation et Simulation Multi Echelle, MSME UMR 8208 CNRS, \\ 5 bd Descartes, 77454 Marne-la-Vallée, France
}

(Received 1 August 2016; revised 20 July 2017; accepted 2 August 2017; published online 29 August 2017)

\begin{abstract}
The purpose of this work is to systematically study the effect of the throat and the pore sizes on the sound absorbing properties of open-cell foams. The three-dimensional idealized unit cell used in this work enables to mimic the acoustical macro-behavior of a large class of cellular solid foams. This study is carried out for a normal incidence and also for a diffuse field excitation, with a relatively large range of sample thicknesses. The transport and sound absorbing properties are numerically studied as a function of the throat size, the pore size, and the sample thickness. The resulting diagrams show the ranges of the specific throat sizes and pore sizes where the sound absorption grading is maximized due to the pore morphology as a function of the sample thickness, and how it correlates with the corresponding transport parameters. These charts demonstrate, together with typical examples, how the morphological characteristics of foam could be modified in order to increase the visco-thermal dissipation effects. (C) 2017 Acoustical Society of America.
\end{abstract}

[http://dx.doi.org/10.1121/1.4999058]

[OU]

Pages: $1130-1140$

\section{INTRODUCTION}

The acoustical properties have been modeled from microstructure for a wide range of materials: granular material such as nickel hollow spheres packings, ${ }^{1}$ open-cell aluminum foams, ${ }^{2}$ perforated closed-cell metallic foams, ${ }^{3}$ lead shot and expanded perlite where the inner structure is modeled as cellular material, ${ }^{4}$ polyurethane foams, ${ }^{5}$ and gypsum foams. ${ }^{6}$

These latter works relate to the identification of microstructural models allowing to capture the main acoustical dissipation mechanisms. They do not include the corresponding parametric study which could lead to an effective improvement of the functional properties. Besides, the transport parameters of sphere and polyhedron packings have been studied for a wide range of porosity using periodic homogenization and self-consistent schemes ${ }^{7}$ with a view to provide analytical approximations of the effective parameters. Although it has been shown that two-dimensional (2D) microstructural models can provide a qualitative insight on the effect of the morphological modifications on the transport properties to understand the sound absorption mechanism, ${ }^{8}$ three-dimensional (3D) results directly applicable to real foam samples are still failing to provide quantitative guidelines for manufacturers.

Moreover, the manufacturing process places constraints on the accessible range of porosities and microscopic parameters (pore size, throat size). Indeed, the processes have their own accuracy and may present slight variations from the

\footnotetext{
a)Electronic mail: fabien.chevillotte@matelys.com

b)Electronic mail: camille.perrot@univ-paris-est.fr
}

normal behavior over a fixed period of time, this phenomenon will be referred to as "process drift." This implies that large computational facilities could be used to study the tolerance of the variation of the target parameters and thus provide guidelines for selecting the appropriate foam morphology.

The 3D idealized periodic microstructural model used in this paper represents a large class of cellular solid foams whose transport properties are of scientific and technological interest. A parametric study is carried out by using this geometrical model to develop a quantitative understanding between pore structure and sound absorbing properties. The following acoustical excitations, normal incidence (NI) and diffuse field (DF), are considered. It is therefore possible to test and compare the simulation results with physical assumptions corresponding, respectively, to impedance tube laboratory measurement and more realistic in situ or reverberant room experiments. However the samples are assumed to have infinite lateral dimensions in this study.

This paper is organized as follows. In Sec. II, classical models to account for visco-thermal dissipation are briefly recalled, the manner to compute the parameters of the models is exposed, and the morphology of the studied foams is presented. Section III is devoted to the parametric analysis. The effects of the throat size on the effective parameters at constant pore size are analyzed, followed by the effects of the pore size at constant throat size. A multi-parametric analysis enables to derive a set of charts that are directly applicable to select the characteristic sizes of the foams that maximize visco-thermal dissipation. This set of charts is then illustrated on practical examples discussed throughout Sec. IV. 


\section{METHODOLOGY}

\section{A. Acoustical modeling of rigid porous media}

The visco-thermal dissipation of acoustical energy through porous media is taken into account by two complex frequency-dependent functions [the dynamic density $\tilde{\rho}_{e q}(\omega)$ and the dynamic bulk modulus $\left.\tilde{K}_{e q}(\omega)\right]$. Acoustic wavelengths are considered larger than the characteristic size of the pores. The decoupling between visco-inertial and thermal effects is also assumed to be valid.

Using semi-phenomenological models, these two functions are analytically derived from macroscopic parameters (time convention $+j \omega t$ ). The most popular semi-phenomenological models are the JohnsonChampoux-Allard (JCA) and the Johnson-ChampouxAllard-Lafarge (JCAL) models. By considering a rigid porous sample saturated by a Newtonian fluid, Johnson et al. ${ }^{9}$ derived a simple yet robust description of frequency-dependent modeling of the visco-inertial effects from macroscopic parameters, namely the open porosity $\phi$, the static airflow resistivity $\sigma$, the high frequency limit of the tortuosity $\alpha_{\infty}$, and the viscous characteristic length $\Lambda$. Subsequently, a relevant description of the frequencydependent thermal dissipation effects was achieved by Champoux and Allard ${ }^{10}$ using an additional parameter, the thermal characteristic length $\Lambda^{\prime}$. Lafarge et al. ${ }^{11}$ improved the dynamic thermal response by introducing the static thermal permeability $k_{0}^{\prime}$. A synthesis of JCA and JCAL models can be found in Ref. 12. Equations of the JCAL model are written as follows.

For visco-inertial effects:

$$
\begin{aligned}
& \tilde{\rho}_{e q}(\omega)=\frac{\rho_{0} \alpha_{\infty}}{\phi}\left[1-j \frac{\omega_{v}}{\omega} \tilde{G}(\omega)\right], \\
& \text { with } \tilde{G}(\omega)=\sqrt{1+\frac{1}{2} j M \frac{\omega}{\omega_{v}}} ; \quad M=\frac{8 k_{0} \alpha_{\infty}}{\phi \Lambda^{2}} ; \\
& \qquad \omega_{v}=\frac{\nu \phi}{k_{0} \alpha_{\infty}} ; \quad \nu=\frac{\eta}{\rho_{0}} .
\end{aligned}
$$

For thermal effects:

$$
\begin{aligned}
& \tilde{K}_{e q}(\omega)=\frac{\gamma P_{0} / \phi}{\gamma-(\gamma-1)\left[1-j \frac{\omega_{t}}{\omega} \tilde{G}^{\prime}(\omega)\right]^{-1}}, \\
& \text { with } \tilde{G}^{\prime}(\omega)=\sqrt{1+\frac{1}{2} j M^{\prime} \frac{\omega}{\omega_{t}}} ; \quad M^{\prime}=\frac{8 k_{0}^{\prime}}{\phi \Lambda^{\prime 2}} ; \\
& \omega_{t}=\frac{\nu^{\prime} \phi}{k_{0}^{\prime}} ; \quad \nu^{\prime}=\frac{\kappa}{\rho_{0} C_{p}} .
\end{aligned}
$$

For classical porous materials, the saturating fluid is air and is taken into account from its density $\rho_{0}$ and compressibility $\gamma P_{0}$. Other parameters are the thermal conductivity $\kappa$, the heat capacity $C_{p}$, the ratio of specific heat $\gamma$, the dynamic viscosity $\eta$, and the atmospheric pressure $P_{0}$.

The characteristic impedance $\tilde{Z}_{c}(\omega)$ and wavenumber $\tilde{k}_{c}(\omega)$ are obtained from the dynamic density and the dynamic bulk modulus

$$
\begin{aligned}
& \tilde{Z}_{c}(\omega)=\sqrt{\tilde{\rho}_{e q}(\omega) \tilde{K}_{e q}(\omega),} \\
& \tilde{k}_{c}(\omega)=\omega \sqrt{\frac{\tilde{\rho}_{e q}(\omega)}{\tilde{K}_{e q}(\omega)}} .
\end{aligned}
$$

The surface impedance of a porous medium of thickness $L$ backed by a hard wall and excited by an oblique incidence plane wave is

$$
\tilde{Z}_{s}(\omega, \theta)=-j \tilde{Z}_{c}(\omega) \frac{k_{c}}{k_{x}} \operatorname{cotg}\left(k_{x} L\right),
$$

with $k_{x}=\sqrt{k_{c}^{2}-k_{t}^{2}}$ the longitudinal wavenumber of the porous medium, $k_{t}=k_{0} \sin (\theta)$ the transverse wavenumber of the porous medium, the acoustical wavenumber of air $k_{0}$, and $\theta$ the angle of incidence in air. In this work, because we computed the dependence of the surface impedance of the model with the angle of incidence, we do not assume that the medium is locally reacting. It is noteworthy that in Eq. (7) the characteristic impedance $\tilde{Z}_{c}$ does not depend on the incidence angle. The computation could be improved by considering that the effective parameters also depend on the incident angle. The overall trends are expected to be similar. This could be the topic of further research.

The sound absorption coefficient $\alpha$ at oblique incidence is calculated by

$$
\alpha(\omega, \theta)=1-\left|\frac{\tilde{Z}_{s}(\omega, \theta) \cos (\theta)-Z_{0}}{\tilde{Z}_{s}(\omega, \theta) \cos (\theta)+Z_{0}}\right|^{2} .
$$

Finally, the sound absorption coefficient $\alpha_{d}$ in a DF is computed by

$$
\alpha_{d}(\omega)=\frac{\int_{\theta_{\min }}^{\theta_{\max }} \alpha(\omega, \theta) \cos (\theta) \sin (\theta) d \theta}{\int_{\theta_{\min }}^{\theta_{\max }} \cos (\theta) \sin (\theta) d \theta},
$$

where $\theta_{\min }$ and $\theta_{\max }$ are the selected DF integration limits, $0^{\circ}$ and $90^{\circ}$ in this work.

\section{B. Hybrid micro-macro method}

Contrary to the direct approach which solves the linearized Navier-Stokes and the heat equations in harmonic regime, the hybrid method relies on approximate but robust semi-phenomenological models (JCA and JCAL). These models are very attractive because they avoid computing numerically in the full frequency range the values of the effective density/bulk modulus. The principle is to solve the local equations governing the asymptotic frequencydependent visco-thermal dissipation phenomena at the microscopic scale. All the macroscopic parameters of interest can be determined from the geometry of the microstructure and only three asymptotic calculations:

(i) The open porosity $\phi$ is defined as the fraction of the interconnected pore fluid volume to the total bulk 
volume of the porous aggregate. A second parameter which is widely used to characterize the macroscopic geometry of porous media is the hydraulic radius, defined as twice the ratio of the total pore volume to its surface area. This characteristic length may also be referred to as the thermal characteristic length $\Lambda^{\prime}$ in the context of sound absorbing materials. ${ }^{10}$ Both of these parameters are estimated by direct spatial integration on the volume and surface elements of the microstructure.

(ii) The static airflow resistivity $\sigma$ (or static viscous permeability $k_{0}=\eta / \sigma$, where $\eta$ is the dynamic fluid viscosity) is computed from the Stokes problem. $9,13,14$ The airflow resistivity is the ratio between the pressure drop and the flow velocity passing through the material. It characterizes the ability of a material to oppose a flow. The static viscous tortuosity $\alpha_{0}$, another transport parameter significant of the viscous flow, ${ }^{15,16}$ might also be computed from the same boundary value problem. The low frequency tortuosity reflects the dispersion of the microscopic velocity field around the mean macroscopic value for quasisteady movements of the viscous fluid flow.

(iii) The viscous characteristic length $\Lambda$ and the high frequency limit of the tortuosity $\alpha_{\infty}$ are calculated using a perfect (non-viscous) incompressible fluid. The potential flow problem is formally identical to the electrical conduction problem for a porous material in which the solid phase is insulating and the saturating fluid is conductive. ${ }^{17}$ They are determined from solutions of the Laplace's equation. $\Lambda$ can be interpreted as a weighted volume-to-surface ratio that accounts for the throat region.

(iv) The static thermal permeability $k_{0}^{\prime}$, also known as the inverse of the trapping constant $\Gamma$ in the context of physical chemistry, was computed by means of a thermal conduction problem where the solid skeleton is considered a thermostat. An additional macroscopic parameter, the static thermal tortuosity $\alpha_{0}^{\prime}$, can be derived from the solution of the same boundary value problem. ${ }^{18}$ It is an analogous parameter to the low frequency tortuosity, where the microscopic temperature field is used instead of the microscopic velocity field.

Practically, this approach is based on finite element method and the study has been carried out using ScalingCell $^{19}$ and FreeFem ++ software. $^{20}$ ScalingCell includes pre- and post-processings and can load meshes obtained using CAD software. Quadratic Lagrange elements are used for the velocity, the temperature, and the electric fields as well as linear Lagrange elements are used for the pressure field. Due to the symmetry of the considered family of microstructures, numerical simulations were performed on a quarter of the total cell. Neumann boundary conditions are used for symmetric boundaries. ${ }^{21,22}$ The uniqueness of the Stokes solution is ensured by forcing the average of the pressure field to zero. Other details related to the computations with appropriate boundary conditions can be found elsewhere ${ }^{5}$ and are not recalled here for brevity.

\section{Selection of the single number rating}

When dealing with a parametric study, the selection of the single number rating is of great importance.

The single number rating $\alpha_{w}$ is commonly used in European countries [ISO 11654 (Ref. 23)] for ranking the sound absorption performances of materials when submitted to a DF excitation. To produce this single number, the measured spectrum is plotted on a graph, and compared against a reference curve [defined in ISO 11654 (Ref. 23)]. The reference curve is moved in 0.05 steps until the total of the unfavorable deviations (measured points on the graph below the reference graph) is as close to 0.1 as possible but not greater than $0.1 . \alpha_{w}$ is the value of the reference curve at $500 \mathrm{~Hz}$ after translation.

Two other single number ratings are commonly used, the sound absorption average (SAA) and the noise reduction coefficient (NRC). The SAA is the average, rounded off to the nearest 0.01 , of sound absorption coefficients in the DF of a material for the 12 one-third octave bands $f_{i}$ from 200 to $2500 \mathrm{~Hz}^{24}$

$$
\mathrm{SAA}=\frac{1}{12} \sum_{f_{i}=200}^{2500} \alpha\left(f_{i}\right) .
$$

The NRC is the rounded average of the sound absorption coefficient for the four octave bands from 250 to $2000 \mathrm{~Hz}$ rounded to the nearest multiple of 0.05 .

The selected single number rating in this study is a SAA over one-third octave bands between 125 and $4000 \mathrm{~Hz}$ $\left(\mathrm{SAA}_{125-4000}\right)$ to enlarge the frequency range of interest. The use of the log scale enables the increase of the low frequency weight. Indeed, the challenge for passive materials is to increase their sound absorbing performance at low frequencies. Here, the parametric study is based on this single number rating with acoustic excitations defined as NI or DF

$$
\mathrm{SAA}_{125-4000}=\frac{1}{16} \sum_{f_{i}=125}^{4000} \alpha\left(f_{i}\right) .
$$

\section{Selection of the representative cell}

There are numerous foaming processes but the principle is always to make growing gas bubbles within a liquid matrix. ${ }^{25}$ In three dimensions, the most simple idealized cell is a regular positioning of spheres with identical sizes. According to the geometric modeling adopted, growing bubbles will tend to interconnect pores and create a network of open pores. This suggests that a body-centered cubic (BCC) arrangement [see Fig. 1(a)] may be proposed as an acceptable approximation of the foam's morphology since it tends toward a tetrakaidecahedron when the pores are growing [see Fig. 1(b)]. The Kelvin's tetrakaidecahedron is well known for modeling high porosity foams such as polymeric cellular structures. ${ }^{26}$ The Kelvin's cell is a tetrakaidecahedron (14-sided polyhedron) with slightly curved faces. Just as in Plateau's experiment, the quadrilateral faces are flat, by symmetry, but the others are curved (with zero total 


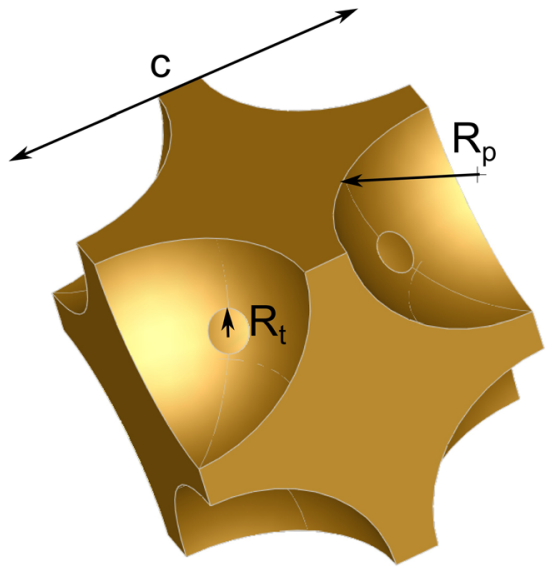

a)

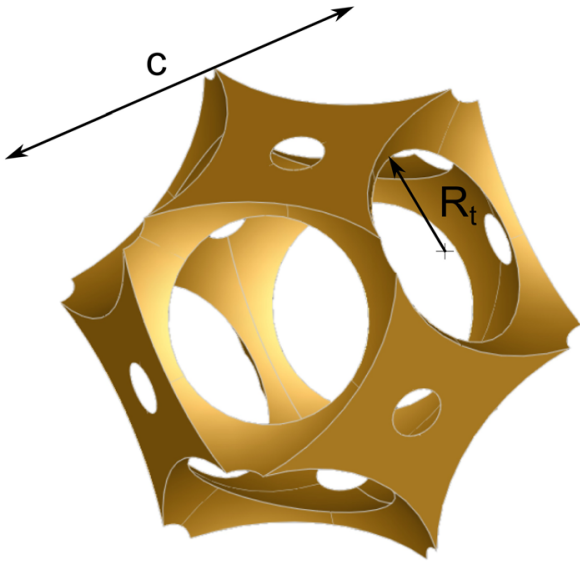

b)
FIG. 1. (Color online) Illustration of cells: (a) Porosity $\phi=0.7(c=571 \mu \mathrm{m}$, $\left.R_{p}=250 \mu \mathrm{m}, R_{t}=37 \mu \mathrm{m}\right)$ (b) Porosity $\phi=0.96\left(c=493 \mu \mathrm{m}, R_{p}=250 \mu \mathrm{m}\right.$, $\left.R_{t}=130 \mu \mathrm{m}\right) . c$ is the width of the cell, $R_{p}$ the pore size, and $R_{t}$ the throat size. curvature). The curved hexagonal faces are almost flat, and Kelvin's tetrakaidecahedron might therefore be described by an approximation, the flat-sided Wigner-Seitz BCC cell. ${ }^{27}$ The Wigner-Seitz cell can be seen as a truncated octahedron. The geometry of the higher density foams does not conform to the classic Kelvin's tetrakaidecahedron model and is described by a spherical shell with circular windows. This allows a continuous common framework of analysis for low and high density foams in which the most important statistical structural parameters which affect acoustical behavior are pore size and throat size.

Knowing the pore size $R_{p}$, the cell size $c$ of the BCC cell, the shortest distance between pores is $c \sqrt{3} / 2$, and using the Pythagorean theorem $R_{p}^{2}=R_{t}^{2}+(c \sqrt{3} / 4)^{2}$, the throat size $R_{t}$ can be calculated for each configuration following this formula:

$$
R_{t}=\sqrt{R_{p}^{2}-\left(c \frac{\sqrt{3}}{4}\right)^{2}}
$$

A systematic application of the model to open-cell foams with porosity $\phi$ in the range 0.7 to 0.99 yields quantitative results linking simple geometrical descriptors such as the pore radius $R_{p}$ and the throat radius $R_{t}$ (i.e., the radius of the narrowest part interconnecting the pore space) with effective transport and sound absorbing properties for various sample thicknesses and different incident excitations. The angle of incidence is taken into account and a robust numerical integration is used to compute the sound absorption coefficient for a DF excitation. This approach also provides information relative to the effect of the manufacturing process variability on the final performance of the product.

\section{PARAMETRIC STUDY}

\section{A. Mesh sensitivity analysis}

A sensitivity analysis is conducted to check the robustness of the numerical method and to ensure that the parametric study is utilizable. The considered cell is a BCC packing with a pore radius $R_{p}$ of $230 \mu \mathrm{m}$, a throat (or window) radius $R_{t}$ of $55 \mu \mathrm{m}$ and a target porosity $\phi_{t}$ of 0.7 . The mesh can be refined by increasing the number of elements $(N)$ per unit length or by increasing the quality of tetrahedrons. The quality factor $Q$ is defined as the ratio of the inscribed sphere radius to minimum edge length. The lower this factor the better the quality of the element. A high factor corresponds to very stretched tetrahedra that can lead to numerical errors, especially to estimate derivative functions. An example of mesh is given in Fig. 2. The mesh sensitivity analysis was also compared to a small variation of the throat size, which is a first order local geometry parameter for predicting the acoustical properties of porous media. Indeed, the throat size is probably the main parameter governing the static airflow resistivity and thus the overall sound dissipation through the porous medium. ${ }^{8}$

The results of the mesh sensitivity analysis are presented in Table I. We found that the macroscopic parameters vary only slightly with typical meshing parameters (less that $1 \%$ ).

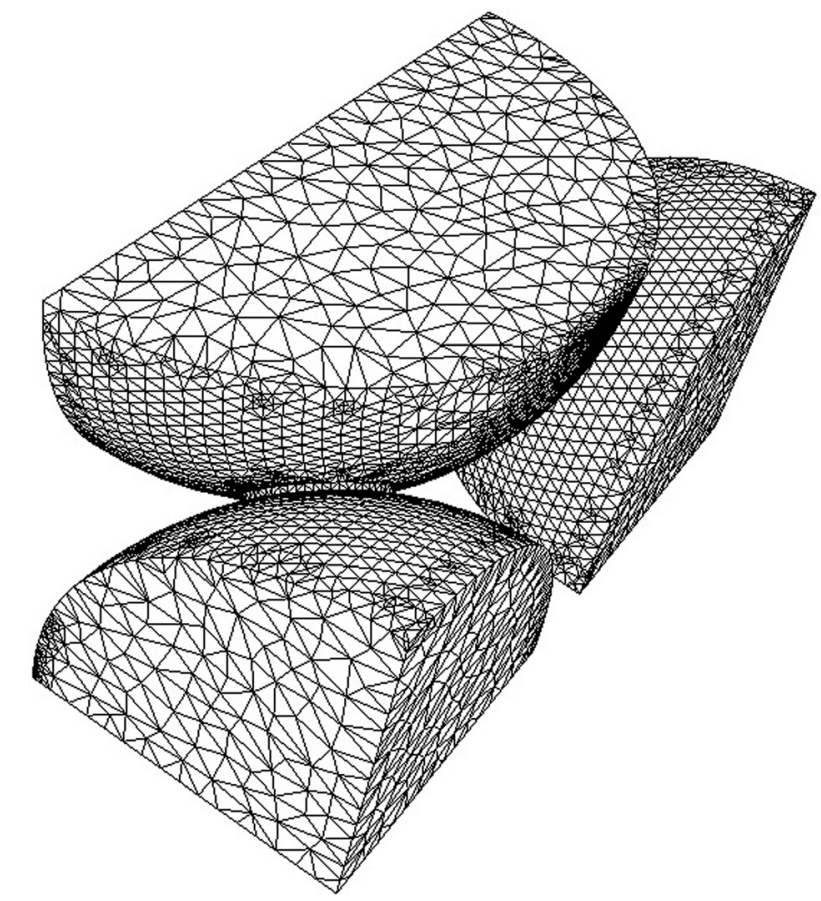

FIG. 2. Example of mesh: $\phi=0.7, R_{p}=0.25 \mathrm{~mm}, R_{t}=0.05 \mathrm{~mm}, N=20$, $Q_{\max }=2$. Due to symmetry of the geometry and the excitation, the computations can be carried out on one-quarter of a single unit cell. 
TABLE I. Mesh sensitivity analysis on the determination of the macroscopic parameters and a comparison with the effect of throat size variation.

\begin{tabular}{|c|c|c|c|c|c|c|c|c|c|c|c|c|c|}
\hline $\begin{array}{l}N \\
\text { Unit }\end{array}$ & $Q$ & $\mathrm{Nb}$. dof & $\begin{array}{c}R_{p} \\
(\mu \mathrm{m})\end{array}$ & $\begin{array}{c}R_{t} \\
(\mu \mathrm{m})\end{array}$ & $\phi$ & $\begin{array}{c}\sigma \\
\left(\mathrm{Nsm}^{-4}\right)\end{array}$ & $\begin{array}{c}\Lambda^{\prime} \\
(\mu \mathrm{m})\end{array}$ & $\begin{array}{c}\Lambda \\
(\mu \mathrm{m})\end{array}$ & $\alpha_{\infty}$ & $\alpha_{0}$ & $\alpha_{0}^{\prime}$ & $\begin{array}{c}k_{0} \\
\left(\times 10^{-10} \mathrm{~m}^{2}\right)\end{array}$ & $\begin{array}{c}k_{0}^{\prime} \\
\left(\times 10^{-10} \mathrm{~m}^{2}\right)\end{array}$ \\
\hline 20 & 2 & 58540 & 230 & 55 & 0.699 & $101541(0 \%)$ & $170(0 \%)$ & $63.5(0 \%)$ & $2.21(0 \%)$ & $3.23(0 \%)$ & $1.40(0 \%)$ & $1.81(0 \%)$ & $25.7(0 \%)$ \\
\hline 30 & 2 & 125406 & 230 & 55 & 0.699 & $100724(0.8 \%)$ & $170(0 \%)$ & $63.3(0.2 \%)$ & $2.2(0.5 \%)$ & $3.22(0.2 \%)$ & $1.40(0 \%)$ & $1.83(0.8 \%)$ & $25.7(0.1 \%)$ \\
\hline 20 & 1.4 & 65016 & 230 & 55 & 0.699 & $100916(0.6 \%)$ & $170(0 \%)$ & $63.4(0.2 \%)$ & $2.18(1 \%)$ & $3.22(0.2 \%)$ & $1.40(0 \%)$ & $1.82(0.6 \%)$ & $25.7(0.1 \%)$ \\
\hline 30 & 1.4 & 128664 & 230 & 55 & 0.699 & $101076(0.5 \%)$ & $170(0 \%)$ & $63.6(0.1 \%)$ & $2.2(0.4 \%)$ & $3.22(0.1 \%)$ & $1.40(0 \%)$ & $1.82(0.5 \%)$ & $25.7(0.1 \%)$ \\
\hline 20 & 1.4 & 66037 & 230 & $56(1.8 \%)$ & 0.699 & $95902(5.6 \%)$ & $170(0.3 \%)$ & $65(2.31 \%)$ & $2.16(2.1 \%)$ & $3.17(1.8 \%)$ & $1.40(0.1 \%)$ & $1.92(5.9 \%)$ & $25.8(0.3 \%)$ \\
\hline 20 & 1.4 & 66935 & 230 & $54(1.8 \%)$ & 0.699 & $106055(4.5 \%)$ & $169(0.3 \%)$ & $62(2.38 \%)$ & $2.22(0.8 \%)$ & $3.27(1.4 \%)$ & $1.40(0.1 \%)$ & $1.73(4.3 \%)$ & $25.7(0.2 \%)$ \\
\hline
\end{tabular}

By contrast, the effect of a small variation of the throat size on the same macroscopic parameters was more significant. This suggests that the method is appropriate to carry out this parametric study.

Indeed, a variation of $1 \mu \mathrm{m}(1.8 \%)$ of the throat radius implies a variation of the same order on the viscous characteristic length $\Lambda$ and on the low and high frequency limits of the tortuosity $\alpha_{0}$ and $\alpha_{\infty}$. Meanwhile, a variation around 5\% is observed in the airflow resistivity. This result is consistent with the fact that for mono-disperse packings the static airflow resistivity is inversely proportional to the square of throat size (assuming independent variables and using the propagation of error formulas, i.e., $\left.\Delta \sigma / \sigma=2 \Delta R_{t} / R_{t}\right)$. Indeed, Johnson et al. ${ }^{9}$ show that the factor $M=8 k_{0} \alpha_{\infty} /$ $\left(\phi \Lambda^{2}\right) \approx 1$ is expected to vary close to one (in Table I, $1.12 \leq M \leq 1.15$ ). Therefore, because $\Lambda$ is proportional to the narrowest passage radius, ${ }^{9} \sigma$ is inversely proportional to the square of the narrowest radius.

The main result of this study is that a small variation of the throat size corresponds to a variation of the macroscopic parameters higher than the error due to the mesh quality.

\section{B. Effect of throat size}

After analyzing the mesh sensitivity, this study is focused on the effect of the throat size on the macroscopic behavior of the material. Some previous parametric studies carried out in two dimensions for fibrous media ${ }^{8}$ and for perforated solid ${ }^{3}$ showed that the most influential microscopic parameter on the sound absorption is the throat size, and that its effect on the airflow resistivity is particularly significant (see Fig. 3 and Table 1 of Ref. 8 and Fig. 7 of Ref. 3).
The BCC cell is used with a pore radius of $230 \mu \mathrm{m}$. The sample thickness $L$ is $25 \mathrm{~mm}$. The throat radius $R_{t}$ varies from 10 to $195 \mu \mathrm{m}$. The influence of the throat size on the sound absorption coefficient is shown in Fig. 3 for NI and DF excitations.

Table II reports the values of the macroscopic parameters and those of the ratings found at NI and DF for each throat radius. Figure 4 displays the relative variations of all the studied macroscopic parameters as a function of the throat radius together with the selected rating in a DF. The variations are expressed as a percentage of the configuration maximizing the SAA rating in a DF $\left(R_{t}=60 \mu \mathrm{m}\right.$, $\left.\mathrm{SAA}_{125-4000}^{\mathrm{DF}}=51 \%\right)$. The throat radius allowing this maximum sound absorption rating will be called the specific throat radius $R_{t}^{\text {spe }}$.

As expected, this analysis shows that the static airflow resistivity $\sigma$ is decreasing when the throat size is increasing. The high frequency limit of the tortuosity $\alpha_{\infty}$ is strongly decreasing as the ratio $R_{p} / R_{t}$ is approaching unity (Table II). Except when the throat size $R_{t}$ tends to the pore size $R_{p}$, the viscous characteristic length $\Lambda$ adopts a value generally close to that of the throat radius $R_{t}$. These results are consistent with what can be found in the literature for simple cubic lattices formed by tubes of size distribution $\mathrm{P}(\mathrm{r})$, where $\mathrm{r}$ is the tube radii. ${ }^{9}$ Indeed, for simple cubic lattices formed by tubes of size distribution $\mathrm{P}(\mathrm{r})$, the viscous characteristic length is on the order of magnitude of the throat size and the static airflow resistivity expected to be inversely proportional to the square of the throat size ${ }^{9}$ since in most cases $M=8 k_{0} \alpha_{\infty} /\left(\phi \Lambda^{2}\right) \approx 1$. When $R_{t}$ tends to $R_{p}$, the solid volume fraction is very small. Furthermore, the surface area of the walls at the fluid/solid interface becomes very small, and the viscous and thermal characteristic lengths increase. Note
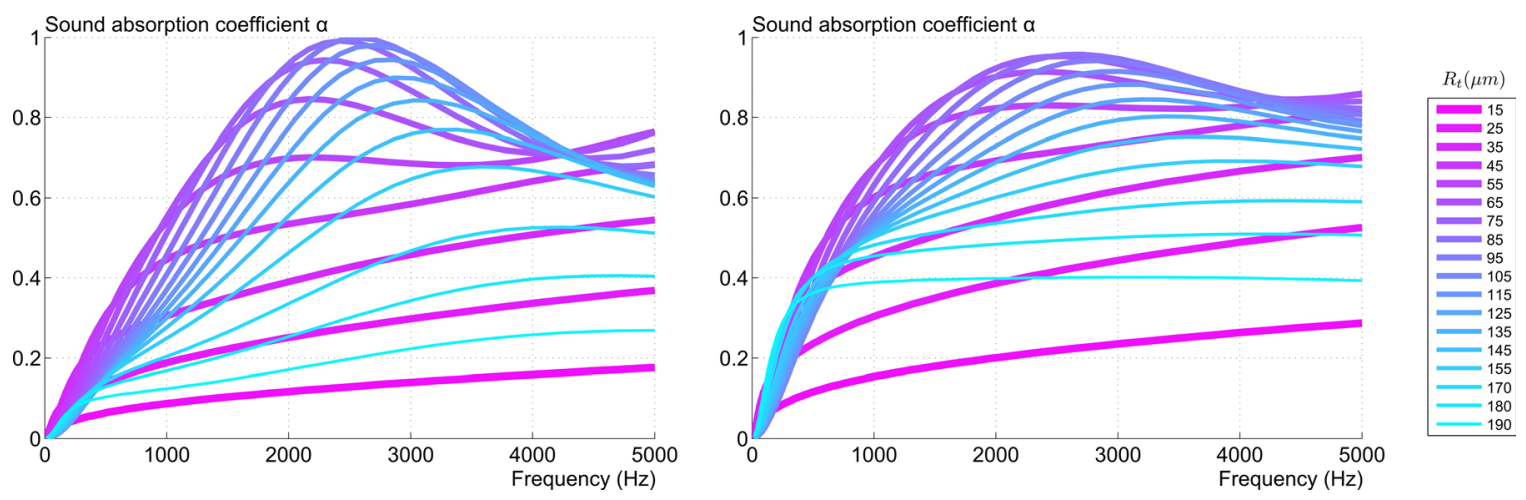

FIG. 3. (Color online) Effect of the throat size on the sound absorption coefficient (left: NI; right: DF). A sample (25 mm thick) with a rigid backing. 
TABLE II. Macroscopic parameters and ratings in NI and DF for different throat radius $R_{t}$.

\begin{tabular}{|c|c|c|c|c|c|c|c|c|c|c|}
\hline$R_{p}(\mu \mathrm{m})$ & $R_{t}(\mu \mathrm{m})$ & $\phi$ & $\sigma\left(\mathrm{Nsm}^{-4}\right)$ & $\Lambda^{\prime}(\mu \mathrm{m})$ & $\Lambda(\mu \mathrm{m})$ & $\alpha_{\infty}$ & $k_{0}^{\prime}\left(\times 10^{-10} \mathrm{~m}^{2}\right)$ & $\operatorname{SAA}_{125-4000}^{N I}(\%)$ & $\mathrm{SAA}_{125-4000}^{D F}(\%)$ & $\alpha_{w}$ \\
\hline 230 & 15 & 0.65 & 6553700 & 154 & 16 & 9.12 & 23 & 8 & 14 & 0.15 \\
\hline 230 & 25 & 0.65 & 1283080 & 156 & 28 & 5.09 & 23 & 18 & 29 & $0.3(\mathrm{H})$ \\
\hline 230 & 35 & 0.65 & 458451 & 158 & 39 & 3.62 & 23 & 28 & 41 & $0.45(\mathrm{H})$ \\
\hline 230 & 45 & 0.65 & 214073 & 162 & 53 & 2.83 & 23 & 35 & 48 & $0.5(\mathrm{H})$ \\
\hline 230 & 55 & 0.65 & 118380 & 167 & 66 & 2.34 & 24 & 40 & 51 & $0.45(\mathrm{MH})$ \\
\hline 230 & 65 & 0.66 & 72887 & 173 & 79 & 2.03 & 24 & 42 & 51 & $0.4(\mathrm{MH})$ \\
\hline 230 & 75 & 0.67 & 48350 & 180 & 90 & 1.8 & 25 & 42 & 49 & $0.4(\mathrm{MH})$ \\
\hline 230 & 85 & 0.67 & 33904 & 189 & 103 & 1.64 & 27 & 41 & 48 & $0.35(\mathrm{MH})$ \\
\hline 230 & 95 & 0.69 & 24917 & 199 & 116 & 1.51 & 29 & 39 & 46 & $0.35(\mathrm{H})$ \\
\hline 230 & 105 & 0.7 & 18862 & 211 & 128 & 1.41 & 31 & 37 & 45 & $0.35(\mathrm{H})$ \\
\hline 230 & 115 & 0.73 & 14675 & 225 & 139 & 1.34 & 34 & 36 & 44 & $0.35(\mathrm{H})$ \\
\hline 230 & 125 & 0.76 & 11628 & 241 & 151 & 1.29 & 39 & 34 & 43 & $0.4(\mathrm{H})$ \\
\hline 230 & 135 & 0.79 & 9289 & 260 & 168 & 1.24 & 45 & 32 & 43 & $0.4(\mathrm{H})$ \\
\hline 230 & 145 & 0.83 & 7173 & 295 & 189 & 1.18 & 54 & 29 & 42 & $0.4(\mathrm{H})$ \\
\hline 230 & 155 & 0.88 & 5442 & 349 & 229 & 1.12 & 67 & 26 & 41 & $0.45(\mathrm{H})$ \\
\hline 230 & 170 & 0.94 & 3494 & 501 & 331 & 1.06 & 98 & 21 & 40 & $0.45(\mathrm{H})$ \\
\hline 230 & 180 & 0.97 & 2515 & 728 & 508 & 1.03 & 132 & 17 & 38 & 0.45 \\
\hline 230 & 190 & 0.99 & 1623 & 1417 & 998 & 1.01 & 197 & 13 & 34 & 0.4 \\
\hline
\end{tabular}

that for $R_{t} \approx R_{p}$, the mechanical strength of the material and the condition of null temperature variations on the material surfaces ${ }^{11}$ become questionable.

Moreover, a specific throat radius can be found for each sample thickness and for each excitation. These specific radii are shown in Fig. 5 for several sample thicknesses in the range $[10-50 \mathrm{~mm}]$. The ranges of radii enabling to reach $95 \%$ of the maximum SAA rating are illustrated with vertical bars for each sample thickness.

More importantly, the specific throat radii are increasing with the sample thickness. This increase looks like a function of the square root of the sample thickness $\sqrt{L}$. The fits of type $\beta \sqrt{L}$ are plotted as dashed lines in Fig. 5. Previous works on perforated solid ${ }^{3}$ and plates $^{28}$ have shown this trend. It has been shown that for thin samples ${ }^{28}$ this specific configuration is obtained by maintaining the airflow resistance $\sigma L$. Considering the shape factor $M=8 \eta \alpha_{\infty} / \phi \sigma \Lambda^{2}$ constant, the viscous characteristic length should be proportional to $\sqrt{L}$ to maintain the resistance $\sigma L$. Unfortunately,

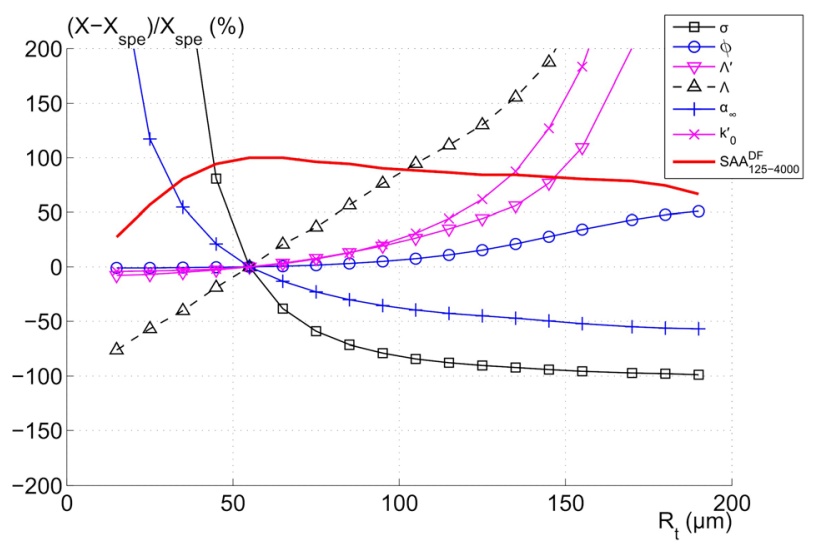

FIG. 4. (Color online) Relative variations of the macroscopic parameters $X$ as a function of the throat radius $R_{t}$, where $X_{\text {spe }}$ are the macroscopic parameters of the specific configuration $R_{t}=R_{t}^{\text {spe }}$. for thicker samples, the relation is not obvious. Thin samples can be seen as resistive screens and their surface impedance only depends on one parameter ${ }^{28}$ since it tends to $\sigma L$. The surface impedance of thicker samples depends on more parameters [see Eqs. (1)-(7)]. The observed correlations between the specific throat radius and the square root of the thickness of the sample provide an indication that the condition of maximizing visco-thermal dissipation may be satisfied by adjusting the resistance $\sigma L$ and not the resistivity itself.

Also worth mentioning, the specific throat radii for DFs are always smaller than the ones corresponding to NI excitation. The range of radii allowing $95 \%$ of the maximum performance widens while increasing the sample thickness. This latter result is important not only for structure-property

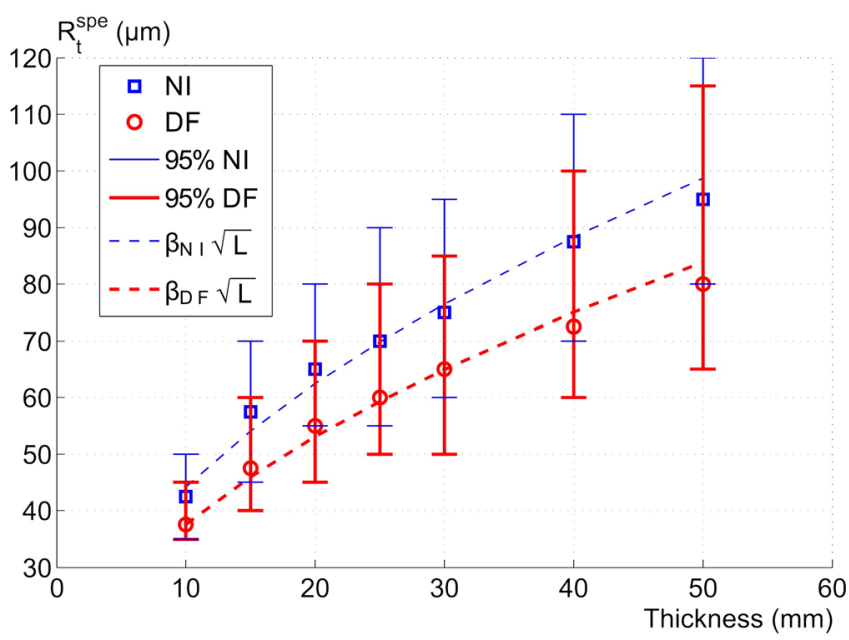

FIG. 5. (Color online) Specific throat radii for NI (square) and DF excitations (circle) as a function of the sample thickness $L$. (Vertical bars: ranges of radii enabling to reach $95 \%$ of the maximum SAA rating for NI and DF; dashed lines: fits of type $\beta \sqrt{L}$ for NI and DF; $\beta_{\mathrm{NI}}=8.8 \times 10^{-4} \mathrm{~m}^{1 / 2}$; $\beta_{\mathrm{DF}}=7.5 \times 10^{-4} \mathrm{~m}^{1 / 2}$ ). 

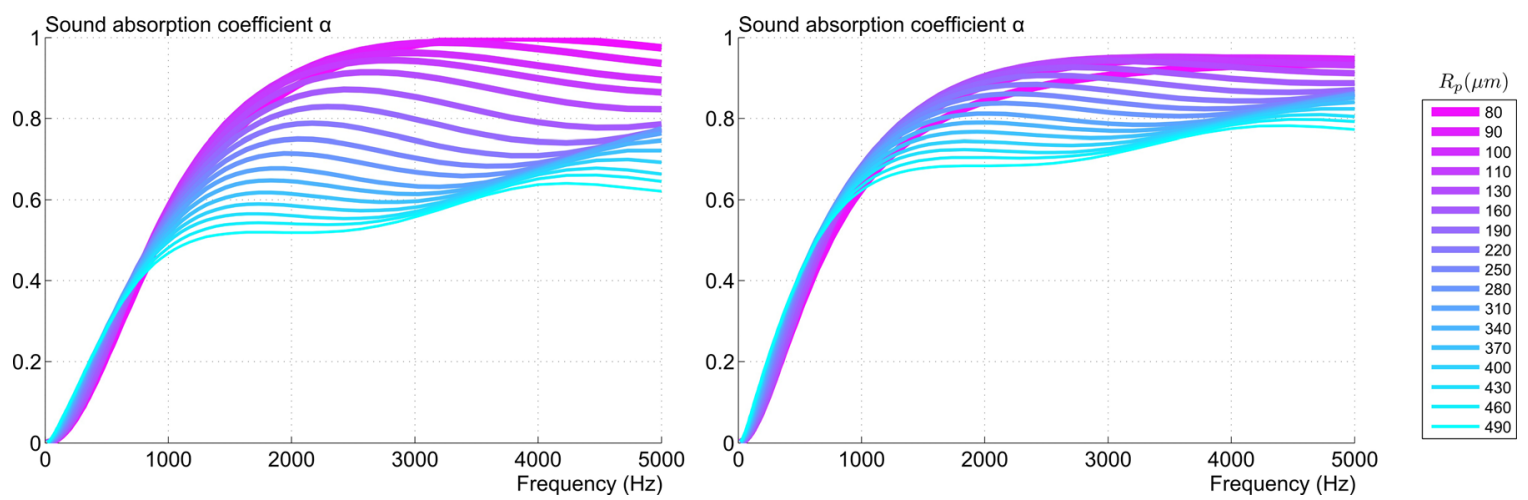

FIG. 6. (Color online) Effect of the pore size on the sound absorption coefficient (left: NI; right: DF). Sample (25 mm thick), rigid backing.

relations but also in terms of manufacturing applications because the smallest thicknesses are more sensitive to modifications of the optimal design parameters. The thicker the sample, the higher the tolerance on the throat size.

\section{Effect of pore size}

The second part of this parametric study is focused on the pore size effect. The sample thickness is $25 \mathrm{~mm}$ with a rigid backing and the initial configuration is the specific one that maximizes the SAA rating in a DF in Sec. III B $\left(R_{p}=230 \mu \mathrm{m}, R_{t}=60 \mu \mathrm{m}, \quad \mathrm{SAA}_{125-4000}^{\mathrm{DF}}=51 \%\right)$. In this study, the pore radius varies from 80 to $490 \mu \mathrm{m}$.

The influence of the pore size on the sound absorption coefficient is shown in Fig. 6 for NI and DF excitations. The pore size has a weaker influence on the overall sound absorption coefficient than the throat size. Moreover, the pore size has a weaker influence with the DF excitation than in NI.

The resultant macroscopic parameters and the corresponding values of the ratings for both the NI and DF excitations are presented in Table III. The reported values vary greatly and are easier to interpret as an overall picture when plotted (Fig. 7). To do so, the variations are expressed with a percentage of the configuration maximizing the SAA rating for both NI and DF $\left(R_{p}=100 \mu \mathrm{m}, R_{t}=60 \mu \mathrm{m}\right.$, $\left.\mathrm{SAA}_{125-4000}^{\mathrm{DF}}=51 \%, \mathrm{SAA}_{125-4000}^{\mathrm{NI}}=46 \%\right)$. The pore radius allowing this maximum sound absorption rating will be called the specific pore radius $R_{p}^{\text {spe }}$.

As it was anticipated thanks to an analysis of the sound absorption curves through Fig. 6, a comparison of Figs. 4 and 7 confirms that the rating is less sensitive to a variation of the pore size in comparison to a variation of the throat size. Based on Fig. 7, it can be seen that two zones are distinguishable, and are separated by the specific configuration maximizing the SAA (this specific configuration is rather a narrow range of specific values corresponding to $\left.100 \leq R_{p}^{\mathrm{spe}} \leq 110 \mu \mathrm{m}\right)$. As it can be seen from the curves in Fig. 7, the thermal length $\Lambda^{\prime}$ and the static thermal permeability $k_{0}^{\prime}$ reach minimal values when the rating achieves its maximum value. This latter correlation has previously been observed in 2D systems. ${ }^{8}$

The evolution of the macroscopic properties might be described according to two distinct behaviors for an increasing pore radius.

- If $R_{p}<R_{p}^{\text {spe }}$, the static airflow resistivity $\sigma$ strongly increases while the viscous and the thermal characteristic lengths $\left(\Lambda, \Lambda^{\prime}\right)$ drastically decrease.

TABLE III. Macroscopic parameters and ratings in NI and DF for different pore radius $R_{p}$.

\begin{tabular}{|c|c|c|c|c|c|c|c|c|c|c|}
\hline$R_{p}(\mu \mathrm{m})$ & $R_{t}(\mu \mathrm{m})$ & $\phi$ & $\sigma\left(\mathrm{Nsm}^{-4}\right)$ & $\Lambda^{\prime}(\mu \mathrm{m})$ & $\Lambda(\mu \mathrm{m})$ & $\alpha_{\infty}$ & $k_{0}^{\prime}\left(\times 10^{-10} \mathrm{~m}^{2}\right)$ & $\mathrm{SAA}_{125-4000}^{N I}(\%)$ & $\mathrm{SAA}_{125-4000}^{D F}(\%)$ & $\alpha_{w}$ \\
\hline 80 & 60 & 0.95 & 26733 & 189 & 126 & 1.05 & 13 & 44 & 48 & $0.4(\mathrm{MH})$ \\
\hline 90 & 60 & 0.87 & 37264 & 133 & 86 & 1.13 & 10 & 46 & 50 & $0.4(\mathrm{MH})$ \\
\hline 100 & 60 & 0.81 & 45627 & 117 & 75 & 1.22 & 9 & 46 & 51 & $0.4(\mathrm{MH})$ \\
\hline 110 & 60 & 0.76 & 50376 & 116 & 73 & 1.29 & 9 & 45 & 51 & $0.4(\mathrm{MH})$ \\
\hline 130 & 60 & 0.71 & 57422 & 120 & 73 & 1.41 & 10 & 44 & 51 & $0.4(\mathrm{MH})$ \\
\hline 160 & 60 & 0.68 & 67571 & 132 & 73 & 1.62 & 13 & 43 & 51 & $0.4(\mathrm{MH})$ \\
\hline 190 & 60 & 0.66 & 77900 & 147 & 72 & 1.86 & 17 & 42 & 51 & $0.4(\mathrm{MH})$ \\
\hline 220 & 60 & 0.66 & 88668 & 164 & 71 & 2.11 & 22 & 41 & 51 & $0.45(\mathrm{MH})$ \\
\hline 250 & 60 & 0.65 & 99746 & 181 & 69 & 2.35 & 28 & 41 & 51 & $0.45(\mathrm{MH})$ \\
\hline 280 & 60 & 0.65 & 110888 & 200 & 72 & 2.63 & 35 & 40 & 51 & $0.45(\mathrm{MH})$ \\
\hline 310 & 60 & 0.65 & 122959 & 218 & 69 & 2.93 & 42 & 39 & 50 & $0.45(\mathrm{MH})$ \\
\hline 340 & 60 & 0.65 & 134401 & 237 & 69 & 3.15 & 51 & 38 & 50 & $0.45(\mathrm{H})$ \\
\hline 370 & 60 & 0.65 & 146981 & 256 & 70 & 3.44 & 60 & 37 & 49 & $0.45(\mathrm{H})$ \\
\hline 400 & 60 & 0.65 & 159744 & 275 & 70 & 3.77 & 70 & 37 & 49 & $0.45(\mathrm{H})$ \\
\hline 430 & 60 & 0.65 & 171320 & 294 & 68 & 4.06 & 80 & 36 & 48 & $0.45(\mathrm{H})$ \\
\hline 460 & 60 & 0.65 & 184615 & 314 & 70 & 4.3 & 92 & 35 & 48 & $0.45(\mathrm{H})$ \\
\hline 490 & 60 & 0.65 & 198762 & 333 & 67 & 4.59 & 104 & 35 & 47 & $0.45(\mathrm{H})$ \\
\hline
\end{tabular}




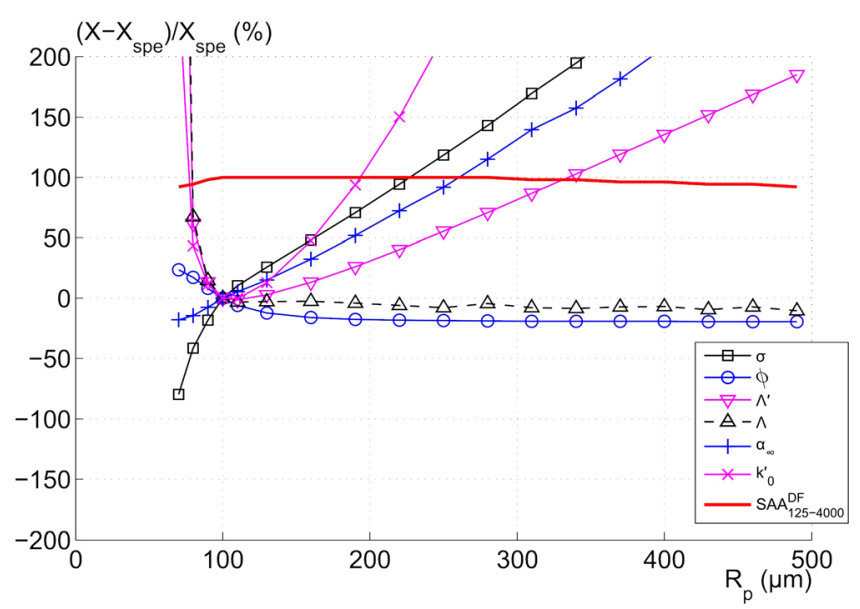

FIG. 7. (Color online) Relative variations of the macroscopic parameters $X$ as a function of the pore radius $R_{p}$, where $X_{\text {spe }}$ are the macroscopic parameters of the specific configuration $R_{p}=R_{p}^{\text {spe }}$.

- If $R_{p}>R_{p}^{\mathrm{spe}}$, the static airflow resistivity $\sigma$, the thermal characteristic length $\Lambda^{\prime}$, and the high frequency limit of the tortuosity linearly increase while the viscous characteristic length $\Lambda$ and the porosity $\phi$ remain nearly constant.

The main influence of the pore size variation on the sound absorption coefficient is an increase of the airflow resistivity and thus a modification of the overall sound absorption level (Fig. 6). Note that the relation $\sigma \approx 8 \eta \alpha_{\infty} /$ $\left(\phi \Lambda^{2}\right)$ is verified $(M \approx 1)$. Looking at Table III for pore radii higher than $160 \mu \mathrm{m}$, the porosity and the viscous characteristic length are almost unchanged and the airflow resistivity increases as the ratio between the pore size and the throat size increases. It is corroborated by an increase of the high frequency limit of the tortuosity. This increase of tortuosity may lead to a modulation of the sound absorption coefficient also called the selectivity effect. ${ }^{8}$ The influence of the throat size is more important because it directly affects the viscous characteristic length and the airflow resistivity can be estimated as in the order of the inverse of the square of the viscous characteristic length.

Additionally, the ratio between the thermal and the viscous characteristic lengths is not constant (Table III), nor equal to three as it can be seen in some inverse characterization procedures. ${ }^{29}$ Therefore, the hypothesis of a constant

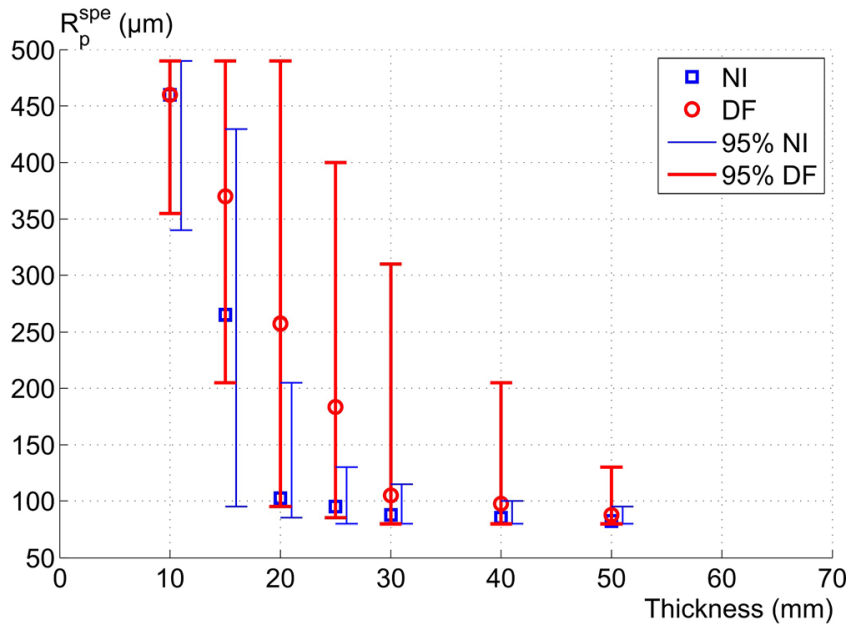

FIG. 8. (Color online) Specific pore radii for NI (square) and DF excitations (circle) as a function of the sample thickness $L$. (Vertical bars: ranges of radii enabling to reach $95 \%$ of the maximum SAA rating for NI and DF.)

$\Lambda^{\prime} / \Lambda$ ratio cannot be made for an accurate characterization of real foam samples by using inversion protocols, especially when structural information on the pore and throat size radii is unavailable.

In fact, a specific pore radius can be found for each sample thickness and for each excitation. These specific pore radii are reported in Fig. 8 for sample thicknesses of 10, 15, $20,25,30,40$, and $50 \mathrm{~mm}$. The ranges of pore radii enabling to reach $95 \%$ of the maximum SAA rating are also calculated and illustrated with the corresponding vertical bars, for each sample thickness.

In contrast to the result on specific throat radii (Fig. 5), the specific pore radii are clearly decreasing with the sample thickness. This result can be explained by the fact that thin samples require a high tortuosity in order to improve the sound absorption. Indeed, in a first approximation, this tortuosity increases the effective thickness by a factor $\sqrt{\alpha_{\infty}}$ and a higher tortuosity value is obtained by increasing the ratio $R_{p} / R_{t}$. The specific pore radii for a DF are always larger than the ones for NI excitation. The range of radii allowing $95 \%$ of the maximum performance is relatively wide for most sample thicknesses.

However, an unambiguous evidence for the nonsymmetrical states of the upper and lower parts of the bars is obtained for most thicknesses. This is in agreement with the
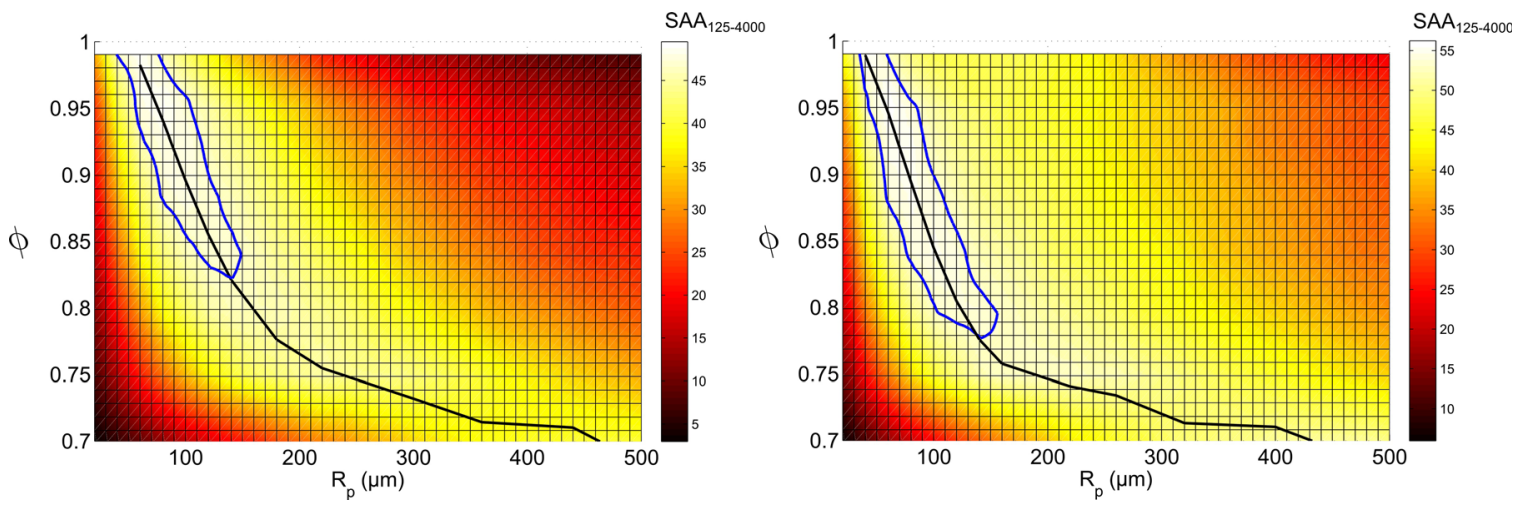

FIG. 9. (Color online) Effect of the open porosity $\phi$ and pore size $R_{p}$ on the sound absorption rating (left: NI; right: DF). Sample (25 mm thick). 


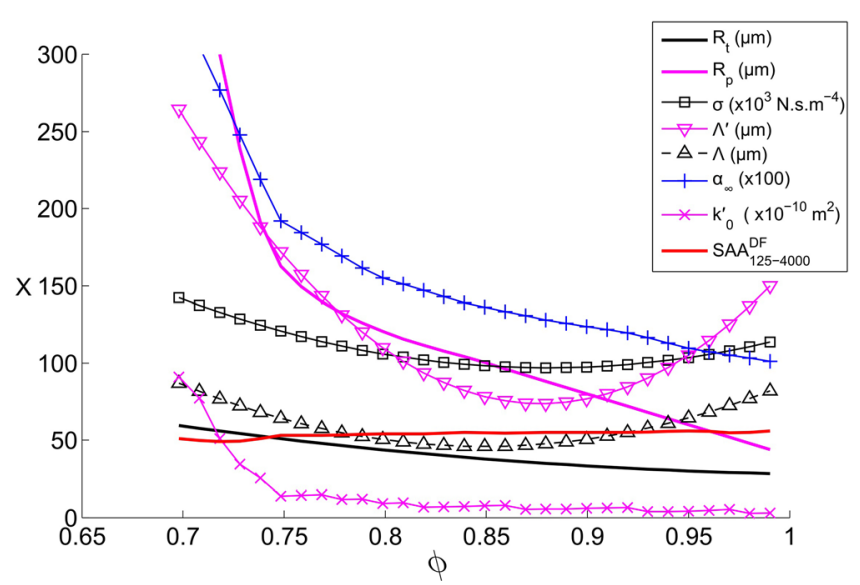

FIG. 10. (Color online) Variations of the microscopic and macroscopic parameters $X$ as a function of the open porosity $\phi$ for configurations maximizing the SAA: $25 \mathrm{~mm}$ thick sample.

two distinct behaviors described above (Fig. 7). This characteristic feature has strong implications on the manufacturing process, especially if one thinks that the phenomenon referred to as "drift" is a normal characteristic of an industry. This implies that it may be advantageous to target a pore size larger than the specific value in order to target the middle of the tolerance zone and to authorize an increase as well as a decrease of the pore size.

More generally, a comparison of the rating curves in Figs. 4 and 7 enables to conclude that the specific pore size is less sensitive to the foaming process drift than the throat size. Indeed, the relative variation of the rating for the pore size is smaller than the relative variation of the rating for the throat size.

\section{Multi-parameter analysis}

The influence of the throat size and the pore size have been independently studied in Secs. III B and IIIC. A full parametric study over $\phi, R_{p}, R_{t}$, and $L$ is carried out in this section.

The SAA rating is analyzed as a function of the porosity $\phi$ and the pore radius $R_{p}$. The resultant 2D maps and the corresponding $\mathrm{SAA}_{125-4000}$ ratings are presented in Fig. 9 for the NI and DF excitations with a thickness of $25 \mathrm{~mm}$. The thick line shows the morphological configurations

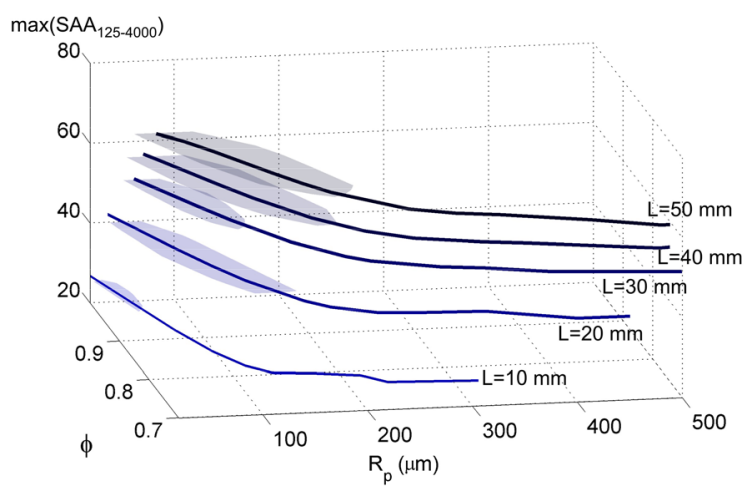

maximizing the sound absorption rating $\left(\mathrm{SAA}_{125-4000}\right)$. The contours enabling $95 \%$ of the maximum performance are also shown in these maps.

The microscopic and macroscopic parameters are given as a function of the open porosity for configurations maximizing the SAA in Fig. 10 (this corresponds to the variation of parameters along the thick line.). The SAA appears to be nearly constant for porosities higher than 0.75 . Indeed, the rating slightly increases from $53 \%$ at $\phi=0.75$ to $56 \%$ at $\phi=0.99$. Meanwhile, the pore size $R_{p}$ largely decreases while the throat size $R_{t}$ decreases as the porosity $\phi$ increases. Clearly, the ratio $R_{p} / R_{t}$ and thus the high frequency limit of the tortuosity $\alpha_{\infty}$ decrease. The static airflow resistivity, the thermal and viscous characteristic lengths show evidence of a minimum in the range of porosities $0.85 \leq \phi \leq 0.90$. In fact, this range of porosity could be correlated with a transition between the moderately porous spherical arrangement and the highly porous tetrakaidecahedron cell (Fig. 1).

The calculated configurations maximizing the sound absorption rating $\left(\mathrm{SAA}_{125-4000}\right)$ for thicknesses between 10 and $50 \mathrm{~mm}$ are also given for the NI excitation (see Fig. 11) and the DF excitation (see Fig. 12). The zones having a rating higher than $95 \%$ of the maximum SAA are also illustrated for each thickness. The value of $95 \%$ is taken for each thickness using the reference of the maximum SAA for the considered thickness.

Finally, the corresponding specific throat radii, maximizing the sound absorption rating, are plotted as a function of the open porosity for thicknesses between 10 and $50 \mathrm{~mm}$ for NI and DF excitations in Fig. 13.

In agreement with what has previously been observed in a low porosity system at $\phi=0.7$ as shown in Sec. III B, the reported specific throat radii are increasing with the sample thicknesses whatever the considered range of open porosities [0.7-0.99]. The reported specific throat radii for the DF are always smaller than those reported for NI excitation but are of a similar order of magnitude for each thickness. More thorough explanations of the use of these graphs will be given in Sec. IV using practical examples.

\section{PRACTICAL EXAMPLES}

As shown in Figs. 11 and 12, the melamine foam, known as a good sound absorber, with parameters $\phi=0.99$,

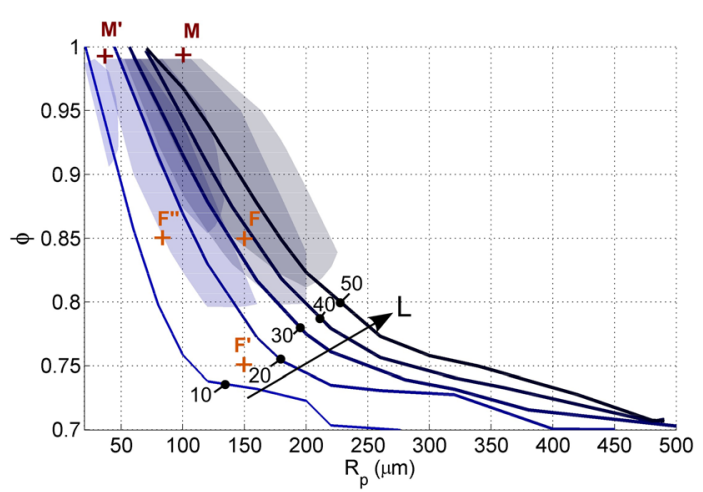

FIG. 11. (Color online) Effect of the open porosity $\phi$ and pore size $R_{p}$ on the sound absorption rating for various sample thicknesses with NI excitation (left: $3 \mathrm{D}$; right: 2D). 

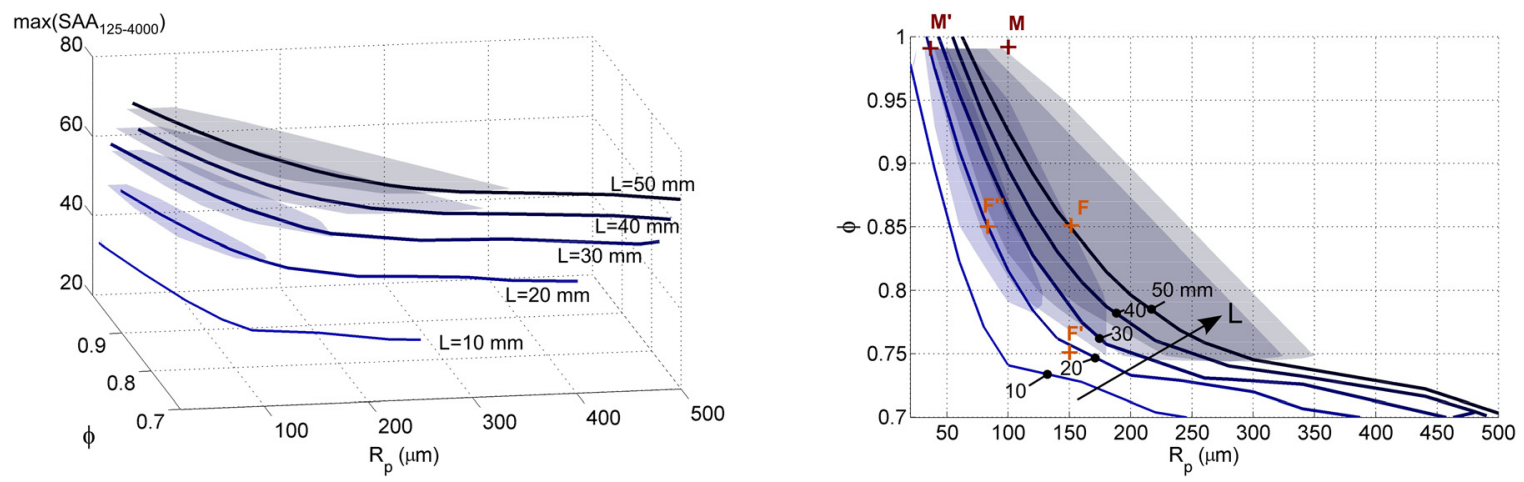

FIG. 12. (Color online) Effect of the open porosity $\phi$ and pore size $R_{p}$ on the sound absorption rating for various sample thicknesses with DF excitation (left: $3 \mathrm{D}$; right: $2 \mathrm{D})$.

$R_{p} \approx 100 \mu \mathrm{m}, R_{t} \approx 50 \mu \mathrm{m}$ has the structure which is optimized for high sound absorption under NI and for thicknesses higher than $30 \mathrm{~mm}$ (marker $\mathrm{M}$ in Figs. 11, 12, and 13, $\left.\mathrm{SAA}_{125-4000}^{\mathrm{NI}}=23 \%, \mathrm{SAA}_{125-4000}^{\mathrm{DF}}=28 \%\right)$. The full list of parameters can be found in Table 13.1 of Ref. 12 and a picture of the morphology can be found in Fig. 3 of Ref. 30. In contrast, the maximum in sound absorption for open cell foams that have a thickness around $10 \mathrm{~mm}$ is directly linked to microstructural characteristic lengths (i.e., pore and throat size) that must be smaller. As it can be seen from Figs. 11 and 13, an improvement of the sound absorption is expected for a structure as defined by a combined reduction of the pore and throat sizes $\left(R_{p} \approx 40 \mu \mathrm{m}, R_{t} \approx 20 \mu \mathrm{m}\right.$, marker $\mathrm{M}^{\prime}$ in Figs. 11, 12, and 13). The single number rating $\mathrm{SAA}_{125-4000}$ increases from $23 \%$ to $27 \%$ in NI and from $28 \%$ to $37 \%$ in the DF.

The second example is provided by foam manufactured such that the following parameters are obtained: $\phi=$ $0.85, R_{p}=150 \mu \mathrm{m}, R_{t} \approx 60 \mu \mathrm{m}$ and $L=20 \mathrm{~mm}$ (marker $\mathrm{F}$ in Figs. 11, 12, and 13, $\left.\mathrm{SAA}_{125-4000}^{\mathrm{DF}}=44 \%\right)$. An analysis of the data reported here reveals that there are three means to improve the sound absorption of this foam:

- The simplest way to improve the sound absorption properties of this foam is to provide it into the thickness corresponding to the best achieved rating at a given pore radius. It is shown in Figs. 11 and 12 that this kind of foam should be used with a thickness of $40 \mathrm{~mm}$ when a NI excitation is considered and a thickness of $50 \mathrm{~mm}$ when a DF is taken as the excitation.

- If the pore size can be reduced to $R_{p} \approx 80 \mu \mathrm{m}$, this would lead to a significant improvement of the sound absorption with a DF excitation while keeping the initial thickness of $20 \mathrm{~mm}$ and the porosity $\phi=0.85$. The throat size would be reduced to $R_{t} \approx 30 \mu \mathrm{m}$ and the single number rating $\mathrm{SAA}_{125-4000}^{\mathrm{DF}}$ would reach $49 \%$ in a DF (marker F' in Figs. 11,12 , and 13).

- Finally, the porosity could be reduced to $\phi=0.75$ to improve the sound absorption while keeping the thickness of $20 \mathrm{~mm}$ and the pore size $R_{p}=150 \mu \mathrm{m}$. The throat size would be reduced to $R_{t} \approx 45 \mu \mathrm{m}$ and the single number rating $\mathrm{SAA}_{125-4000}^{\mathrm{DF}}$ would reach $48 \%$ in a DF (marker F' in Figs. 11, 12, and 13).

\section{CONCLUSION}

The computations of transport and sound absorbing properties of 3D open cell foams combined with systematic modifications of their local characteristic sizes could lead to new insights on the morphologies to be obtained in order to achieve the maximal performance.

As a first step along these lines, we further analyzed the effect of the throat size on the macroscopic parameters governing sound absorption (Fig. 3). The numerical estimates of the transport and sound absorbing properties obtained from a
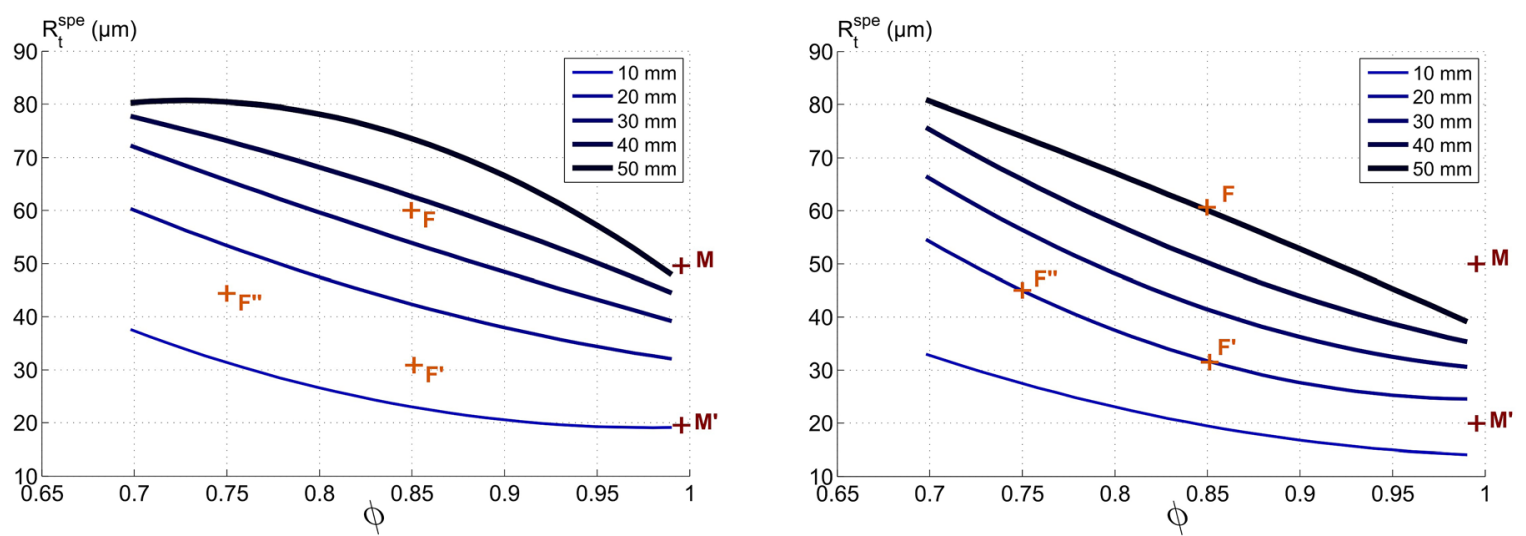

FIG. 13. (Color online) Specific throat radii as a function of the open porosity for various sample thicknesses (left: NI; right: DF). 
3D model in a bottom-up approach put forward results which can be used to simultaneously follow the evolution of macroscopic parameters with the throat size variations, as illustrated in Fig. 4. The throat size is the key morphological parameter for controlling the sound absorption. A 35\% improvement of SAA rating in a DF can be expected for a $25 \mathrm{~mm}$-thick sample (see Table II) but the potential gain increases with the thickness of the material.

These calculations at different sample thicknesses clearly reveal that the optimal throat size increases with the sample thickness of the porous material with a lower value in a DF when compared to NI (Fig. 5). The increase of the specific throat radius with the sample thickness reveals that the visco-thermal dissipation may be maximized by adjusting the airflow resistance $\sigma L$. The airflow resistivity $\sigma$ should therefore be decreased for thicker samples. Because the specific throat radii tolerance maximizing the SAA increases along with the sample thickness, this also suggests that applications targeting small sample thicknesses require a more elaborated control of the manufacturing process drift.

Controlling pore and throat size modifications is a useful strategy for engineering the sound absorption spectrum of open cell foam. However, the emphasis here is on the different possible strategies leading to a significantly enhanced SAA $\left(\mathrm{SAA}_{125-4000}\right)$. Indeed, the effects of the pore size modifications discussed above (Figs. 6, 7, and 8) can be seen as a supplementary morphological parameter along with the throat size, the porosity, and the sample thickness of the foam to map the typical features of the cellular materials (Figs. 9 and 10). This successful bottom-up approach opens extensive opportunities for the analysis of their transport and sound absorbing properties (see Sec. IV for practical examples) and for the manufacturing of porous samples with enhanced performance by following the guidelines provided in this paper (e.g., Figs. 11, 12, and 13).

\section{ACKNOWLEDGMENTS}

Emmanuel Guillon from LafargeHolcim as well as Luc Jaouen and François-Xavier Bécot from Matelys are gratefully acknowledged for fruitful discussions.

${ }^{1}$ S. Gasser, F. Paun, and Y. Bréchet, "Absorptive properties of rigid porous media: Application to face centered cubic sphere packing," J. Acoust. Soc. Am. 117(4), 2090-2099 (2005).

${ }^{2}$ C. Perrot, F. Chevillotte, and R. Panneton, "Dynamic viscous permeability of an open-cell aluminum foam: Computations versus experiments," J. Appl. Phys. 103, 024909 (2008).

${ }^{3}$ F. Chevillotte, C. Perrot, and R. Panneton, "Microstructure based model for sound absorption predictions of perforated closed-cell metallic foams," J. Acoust. Soc. Am. 128(4), 1766-1776 (2010).

${ }^{4} \mathrm{R}$. Venegas and O. Umnova, "Acoustical properties of double porosity granular materials," J. Acoust. Soc. Am. 130(5), 2765-2776 (2011).

${ }^{5}$ C. Perrot, F. Chevillotte, M. T. Hoang, G. Bonnet, F.-X. Bécot, L. Gautron, and A. Duval, "Microstructure, transport, and acoustic properties of open-cell foam samples: Experiments and three-dimensional numerical simulations," J. Appl. Phys. 111, 014911 (2012).
${ }^{6}$ F. Chevillotte, C. Perrot, and E. Guillon, "A direct link between microstructure and acoustical macro-behavior of real double porosity foams," J. Acoust. Soc. Am. 134(6), 4681-4690 (2013).

${ }^{7}$ C. Boutin and C. Geindreau, "Periodic homogenization and consistent estimates of transport parameters through sphere and polyhedron packings in the whole porosity range," Phys. Rev. E 82, 036313 (2010).

${ }^{8}$ C. Perrot, F. Chevillotte, and R. Panneton, "Bottom-up approach for microstructure optimization of sound absorbing materials," J. Acoust. Soc. Am. 124(2), 940-948 (2008).

${ }^{9}$ D. L. Johnson, J. Koplik, and R. Dashen, "Theory of dynamic permeability and tortuosity in fluid-saturated porous media," J. Fluid Mech. 176, 379-402 (1987).

${ }^{10}$ Y. Champoux and J. F. Allard, "Dynamic tortuosity and bulk modulus in air-saturated porous media,” J. Appl. Phys. 70, 1975-1979 (1991).

${ }^{11}$ D. Lafarge, P. Lemarinier, J. F. Allard, and V. Tarnow, "Dynamic compressibility of air in porous structures at audible frequencies," J. Acoust. Soc. Am. 102, 1995-2006 (1997).

${ }^{12}$ J. F. Allard and N. Atalla, "Propagation of sound in porous media," in Modeling Sound Absorbing Materials, 2nd ed. (Wiley, Chichester, United Kingdom, 2009), Chap. 5, 358 pp.

${ }^{13}$ J. L. Auriault, L. Borne, and R. Chambon, "Dynamics of porous saturated media, checking of the generalized law of Darcy," J. Acoust. Soc. Am. 77(5), 1641-1650 (1985).

${ }^{14}$ J.-L. Auriault, C. Boutin, and C. Geindreau, Homogenization of Coupled Phenomena in Heterogeneous Media (Wiley-ISTE, London, United Kingdom, 2009), pp. 197-226.

${ }^{15}$ A. N. Norris, "On the viscodynamic operator in Biot's equations of poroelasticity,” J. Wave Mat. Interact. 1, 365-380 (1980).

${ }^{16}$ S. R. Pride, F. D. Morgan, and A. F. Gangi, "Drag forces of porousmedium acoustics," Phys. Rev. B 47, 4964-4978 (1993).

${ }^{17}$ R. J. S. Brown, "Connection between formation factor for electrical resistivity and fluid-solid coupling factor in Biot's equations for acoustic waves in fluid-filled porous media," Geophys. 45, 1269-1275 (1980).

${ }^{18} \mathrm{D}$. Lafarge, "Propagation du son dans les matériaux poreux à structure rigide saturés par un fluide viscothermique" ("Sound propagation in rigid porous media saturated by a viscothermal fluid"), Ph.D. dissertation, Université du Maine, 1993.

${ }^{19}$ http://scalingcell.matelys.com/ (Last viewed 20 July 2017).

${ }^{20}$ F. Hecht, "New development in FreeFem++," J. Numer. Math. 20(3-4), 251-265 (2012).

${ }^{21}$ V. Tarnow, "Airflow resistivity of models of fibrous acoustic materials," J. Acoust. Soc. Am. 100, 3706-3713 (1996).

${ }^{22}$ R. Hilfer and C. Manwart, "Permeability and conductivity for reconstruction models of porous media,” Phys. Rev. E 64, 021304 (2001).

${ }^{23}$ ISO 11654, "Acoustics-Sound absorbers for use in buildings-Rating of sound absorption" (International Organization for Standardization, Geneva, Switzerland, 1997).

${ }^{24}$ ASTM C423, "Standard test method for sound absorption and sound absorption coefficients by the reverberation room method" (American Society for Testing and Materials, Philadelphia, PA, 2002).

${ }^{25}$ A. Cunningham and N. C. Hilyard, "Physical behaviour of polymeric foams-An overview," in Low Density Cellular Plastics: Physical Basis of Behaviour, edited by N. C. Hilyard and A. Cuningham, 1st ed. (Chapman \& Hall, London, 1994), Chap. 1, pp. 1-21.

${ }^{26} \mathrm{D}$. Weaire and S. Hutzler, The Physics of Foams (Oxford University Press, Oxford, 1999), 264 pp.

${ }^{27}$ D. Weaire, "Kelvin's foam structure: A commentary," Philos. Mag. Lett. 88, 91-102 (2008).

${ }^{28} \mathrm{~F}$. Chevillotte, "Controlling sound absorption by an upstream resistive layer," Appl. Acoust. 73, 56-60 (2012).

${ }^{29}$ Z. E. A. Fellah, F. G. Mitri, M. Fellah, E. Ogam, and C. Depollier, "Ultrasonic characterization of porous absorbing materials: Inverse problem,” J. Sound Vib. 302, 746-759 (2007).

${ }^{30}$ L. Jaouen, A. Renault, and M. Deverge, "Elastic and damping characterizations of acoustical porous materials: Available experimental methods and applications to a melamine foam," Appl. Acoust. 69, 1129-1140 (2008). 